\title{
La Crisis Argentina: Su Impacto en las Relaciones Bilaterales Argentino-Brasileñas y Sobre el MERCOSUR.
}

\author{
Raúl Bernal-Meza ${ }^{1}$
}

\begin{abstract}
Resumen
Este trabajo trata sobre la crisis Argentina como una crisis terminal, de un tipo particular de organización del Estado y de sus relaciones con la sociedad; del fracaso de las estrategias nacionales de desarrollo seguidas desde 1860 y de su modelo de inserción internacional. Desde marzo de 1976 con el golpe de Estado y los sucesivos gobiernos democráticos ( Alfonsín, Menem (1989-1999), De la Rúa (1999-2001) y Duhalde) se impuso en la Argentina una nueva alianza de grupos de poder militar y financiero cuyo resultados fueron la destrucción de gran parte de la estructura productiva, el aumento de la deuda externa (3.000 para 50.000 millones de dólares), la implantación de un modelo neoliberal y la continuación y acelereración del proceso de expoliación y transferencia de recursos. En este sentido, frente al fracaso del modelo neoliberal, el derrumbe económico y la crisis institucional, ética y moral. Los nuevos políticos como Roberto Lavagna están en la obligación de encontrar una salida a la crisis y el pueblo argentino de una "esperanza" que consiste en la revisión de los procesos de integración regional, del Mercosur y de las relaciones bilaterales con Brasil.
\end{abstract}

Palabras-clave: Crisis Argentina, MERCOSUR, Relaciones Bilaterales Argentina-Brasil.

\section{Resumo}

O trabalho trata sobre a crise Argentina como uma crise terminal, de um tipo particular de organização do Estado e de suas relações com a sociedade; do fracasso das estratégias nacionais de desenvolvimento seguidas desde 1860 e de seu modelo de inserção internacional. Desde março de 1976 com o golpe de Estado e os sucessivos governos democráticos (Alfonsín, Menem (1989-1999), De la Rúa (1999-2001) y Duhalde) Empossou-se na Argentina uma nova aliança de grupos de poder militar e financeiro cujos resultados foram a destruição de grande parte da estrutura produtiva, o aumento da devida externa (3.000 para 50.000 milhões de dólares), a implantação de modelo neoliberal e a continuação e aceleração do processo de espoliação e transferência de recursos. Neste sentido, frente ao fracasso do modelo neoliberal, o derrube econômico, a crise institucional, ética e moral. Os novos políticos como Roberto Lavagna têm a obrigação de encontrar uma saída à crise e o povo argentino a "esperança" que consiste na revisão dos processos de integração regional, do Mercosul e das relações bilaterais com o Brasil.

Palavras-clave: Crise Argentina, MERCOSUL, Relações Bilaterais Argentina-Brasil.

1 Licenciado en Ciencias Sociales y Políticas (Université de la Sorbonne); Master en Relaciones Internacionales (FLACSO-Argentina); Master en Economía Política (Sorbonne); Doctor en Sociología (Sorbonne-Universidad Católica Argentina). Profesor Titular de Relaciones Internacionales de la Universidad Nacional del Centro de la Provincia de Buenos Aires y Profesor de la Universidad de Buenos Aires. Director del Centro de Estudios de las Relaciones Internacionales de América Latina, CERIAL, Mendoza, Argentina. Profesor Visitante de la Universidades de São Paulo-USP y Brasilia. E-mail: bernalmeza@hotmail.com ; cerial@infovia.com.ar. 


\section{Introducción}

Como es de conocimiento internacional, la Argentina se encuentra inmersa en la más grave crisis de su historia como nación políticamente independiente. Por primera vez, se trata de una situación que afecta lo económico, lo social, lo político y lo institucional. Nuestra hipótesis es que se trata de una crisis prácticamente terminal de un tipo particular de organización del Estado y de sus relaciones con la sociedad; de las fracasadas estrategias nacionales de desarrollo seguidas desde 1860 y de su modelo de inserción internacional (Bernal-Meza, 2001; 2001 ${ }^{\text {a }}$. Como corolario de ello, las presunciones que entre fines del siglo XIX y comienzos del XX se hicieron sobre el destino exitoso de la Argentina, resultaron lo opuesto de aquello que se esperaba.

El país, gracias fundamentalmente a la miopía, egoísmo y falta de espíritu nacional de sus clases dirigentes (políticas, empresarias, militares, sindicales, episcopales, etc.) fracasó en su camino al desarrollo y hoy se encuentra a las puertas de un oscuro desenlace. Aún más, para muchos analistas, nacionales e internacionales, resulta sorprendente que a esta altura de la situación, el país no se haya incendiado por los cuatro costados; que los trabajadores de todo nivel no hayan declarado una huelga general e indefinida exigiendo la recomposición de sus salarios que se licuaron como consecuencia de la devaluación del dólar (de un tipo de cambio $1 \mathrm{u} \$ \mathrm{~s}=1$ peso, al 10 de diciembre de 2001, a $1 \mathrm{u} \$ \mathrm{~s}=3,40$ pesos en el momento de escribirse este documento) y de la inflación acumulada en los últimos 90 días (que alcanza cifras cercanas al 60\%). La Argentina está al borde de la hiperinflación, con una crisis social y económica que ha lanzado en los últimos 12 años de neoliberalismo a más del $30 \%$ de su población a la pobreza extrema, cifra que se agregó a aquella del $25 \%$ estructuralmente histórica. Un simple dato de la realidad diaria pone en evidencia la situación crítica de la clase media. Según la prensa de Buenos Aires, un 12,5\% de los teléfonos de residencias tienen algún tipo de interrupción en el servicio, derivado de la falta de pago del abono bimestral; de ellos, ochocientas mil líneas telefónicas, que corresponden al $10 \%$ de todas las líneas habilitadas en el país están actualmente con interrupción total del servicio $^{2}$.

\footnotetext{
${ }^{2}$ Cfr. Diario La Nación, Buenos Aires, 6 de mayo de 2002.
} 
La economía productiva, así como su sistema financiero-bancario y el crédito están destruidos. Las instituciones van por el mismo camino; pero lo más grave, más grave aún que el descrédito de los políticos y gobernantes es la falta de seguridad jurídica. Así, con 200.000 millones de dólares de deuda externa; una fuga de capitales que en los últimos diez años alcanzó la cifra de 150.000 millones de dólares; la expoliación que la alianza entre los grupos de poder económico-financieros y los partidos Peronista, Radical y Frepaso hicieron sobre el ahorro de las personas comunes, simples trabajadores y clases medias, que le permitió a los poderosos apropiarse de 68.000 millones (entre 46.000 millones de dólares y 22.000 millones de pesos) en los últimos meses, al mismo tiempo que desde el gobierno de Duhalde se beneficiaba a los grandes grupos de poder económico-financiero con la pesificación ${ }^{3}$ de sus deudas, con lo cual lograron apropiarse de otros 22.000 millones de dólares entre febrero y marzo de este año, el pueblo, la sociedad civil, no tiene poderes que lo protejan y defiendan, porque no existe cumplimiento de los principios enunciados en la Constitución, que aseguraban el respeto a los bienes personales y el derecho a recurrir a la justicia en defensa de ellos. Pero es parte de la política de gobierno que no se respeten. La denegación de Justicia proviene desde el Poder Ejecutivo a través de Decretos inconstitucionales en su forma y contenido.

Entre todas las violaciones a los principios, garantías y derechos, el que se ha visto más afectado, es el principio de igualdad. Y la gravedad proviene de la acción consciente del gobierno, a través de un actuar negligente y falto de decisión. A todas luces, es evidente que a quienes se ha querido beneficiar ha sido a esos grandes grupos de interés.

Pero el factor que más afecta la esperanza del pueblo argentino es la impunidad de los poderosos, aquellos que forman parte de la nueva alianza de poder. Increíblemente, la situación arriba descrita no tiene hoy responsables que estén siendo juzgados por los Tribunales ${ }^{4}$; la Argentina está en la debacle y nadie es responsable...

\footnotetext{
${ }^{3}$ Conversión compulsiva a pesos argentinos a una tasa de cambio de $1 \mathrm{u} \$ \mathrm{~s}=1,40$ pesos, emitida por decreto gubernamental de Duhalde, de los depósitos bancarios en moneda extranjera y de las deudas en la misma moneda.

${ }^{4}$ El único funcionario, el ex ministro Domingo Cavallo, procesado y con prisión preventiva, lo está por un delito de exportación ilegal de material bélico, realizada bajo la gestión del presidente Menem.
} 
Sin embargo, nadie debería llevarse a error: nada de lo que ha ocurrido en la Argentina hubiera pasado sin la connivencia de los poderes públicos llamados a ejercer control sobre los actos de gobierno. La democracia argentina, tan duramente recuperada, no ha servido para salvar a la República de los lobos que la atacan.

\section{El fracasado destino como país}

En marzo de 1976 un golpe de Estado, último de la serie iniciada en 1930, impuso una nueva alianza de grupos de poder. Asociados al poder militar, el sector financiero se constituyó a la cabeza del poder económico del país, desplazando de ese sitio al sector industrial, hecho que significaría el inicio de la destrucción de gran parte de la estructura productiva y del empleo genuino. Bajo su hegemonía, un nuevo grupo económico, heredero de las prebendas de la política (“compre nacional”) de la patria contratista ${ }^{5}$ consolidó en los años siguientes un modelo político y económico que sería la expresión del más salvaje neoliberalismo y que se extendería hasta el presente.

Lo que cambió con la llegada de la democracia, en 1983, fue la constitución política de esa alianza. Durante los años de Alfonsín, un período de políticas heterodoxas intentó enfrentar a estos grupos de poder, pero fracasó, pagando con su retiro anticipado del gobierno su intención de controlar a los mismos.

Para cuando asumió Alfonsín, la deuda externa que en 1975 era de 3.000 millones de dólares había pasado a casi 50.000 millones (diciembre de 1983) y correspondía al 70\% del PBI. La relación entre los intereses pagados de la deuda externa y las exportaciones pasó del 8\% al 60\%, en el mismo período. Sólo en 1983 los precios al consumidor habían subido más del 400\%, y la inflación estaba descontrolada, con una tasa anual superior al 300\%. Las reservas estaban casi agotadas (Treber, 1987), y existían 20.000 millones de

\footnotetext{
${ }^{5}$ Nombre con el cual la sociedad argentina identificó la alianza entre las grandes empresas, los grupos empresarios y la burocracia estatal, incluyendo la dirigencia política de gobierno y la dirigencia sindical, que se distribuyó importante parte de la renta nacional, gracias a los negocios con la provisión de bienes y servicios a las reparticiones y organismos públicos del Estado.
} 
dólares de atrasos en los pagos externos (Rapoport, 2000). Para el año de inicio del Programa de Integración Argentino-Brasileño (PICAB), el "Plan Austral", con que el gobierno de Alfonsín había intentado enfrentar la crisis y la primera de sus hiperinflaciones a través de un programa económico heterodoxo (una nueva moneda; congelamiento de salarios; aumento y luego congelamiento de precios de tarifas públicas; reducción de las tasas de interés reguladas; devaluación y luego congelamiento del tipo de cambio y aumento de impuestos a las exportaciones) había fracasado. Comenzaba a partir de aquí el camino de retorno hacia las políticas de ajuste estructural.

La llegada al gobierno de Carlos Menem (1989-1999) iniciaría la segunda etapa, aún más radicalizada, del extremismo neoliberal, que seguirían De la Rúa (1999-2001) y ahora Duhalde. La alianza de poder encontró nuevos actores políticos. Durante la gestión Menem ésta fue constituida en gran parte por el partido Justicialista y el sector sindical; mientras que bajo la gestión de De la Rúa, lo constituirían la derecha del radicalismo y parte del peronismo. Actualmente, desde la salida de De la Rúa, la alianza política que está asociada a los grupos financieros, las corporaciones y grandes empresas privatizadas, la banca, los servicios y las empresas transnacionales, está formada por el Justicialismo, gran parte del Radicalismo y gran parte del Frepaso. Un simple dato permite confirmar estos hechos: la coalición Partido Radical-Frepaso (que llevó a De la Rúa al gobierno) dio a Duhalde los instrumentos políticos (autonomía para dictar "Decretos de Necesidad y Urgencia”) y aprobado las leyes con los cuales éste ha continuado y acelerado el proceso de expoliación y transferencia de recursos hacia los grandes grupos de poder. De esta forma, los partidos políticos de la oposición claudicaron nuevamente en su compromiso de defender los intereses de sus votantes, viciando de sentido su propia existencia. Hoy, aliados al partido Justicialista y formando parte del gobierno, no tienen validez ética ni moral para representar a nadie.

La sustitución del sector económico-industrial entonces hegemónico por el sector financiero, impulsada por la dictadura militar en 1976, no fue un hecho fortuito ni casual. Obedeció a una definida estrategia que alteraba sustancialmente las políticas de inserción internacional seguidas hasta entonces por el país. En efecto, sus grupos de poder, que veían 
a la Argentina como un país pequeño en el escenario internacional dieron prioridad a la inserción en el mercado internacional de capitales, a partir de la evidencia de que esos flujos eran determinantes de su ciclo económico (Baumann, 2001), adoptando entonces políticas adecuadas a ese objetivo. El plan de "Convertibilidad", diseñado e implementado por Cavallo, bajo la gestión de Menem, a mediados de los años 90, que ancló el peso a una paridad irreal 1 a 1 respecto del dólar, sería un elemento clave de esta nueva estrategia, que De la Rúa mantendría también bajo su gestión.

La historia de fracasos se remontaba a la declinación del exitoso período de inserción primaria o agroexportadora (1880-1916), que se cerraría con el primer golpe de Estado, en 1930. El modelo agroexportador asoció el crecimiento nacional al sistema económico, comercial y financiero de Gran Bretaña. Los avances alcanzados bajo el mismo crearon la Argentina moderna y permitieron un desarrollo económico que registró altos porcentajes en el PBI per cápita, cercanos al de las economías europeas más desarrolladas de la época, período en el cual el país llegaría a representar el 3\% del comercio mundial (Ferrer, 1998; Rapoport, 2000).

En 1913 el ingreso per cápita argentino era el 50\% del norteamericano y el 80\% del promedio del conjunto de las economías industrializadas de Europa. A partir de la primera Guerra Mundial el progreso se detuvo. La dinámica del crecimiento, sostenida por la demanda internacional de carnes, cueros y granos puso entonces en evidencia la fragilidad de un modelo orientado al exterior y basado en ventajas comparativas estáticas. La caída del sistema económico mundial británico arrastraría a la Argentina a la que entonces pareció la más grave crisis desde su unificación nacional (1853). A partir de allí y sobre la base de un promisorio desarrollo industrial (pero que no fue asociado al eslabonamiento horizontal y vertical respecto de las dotaciones naturales de recursos), alcanzado bajo el período agroexportador, el país inició la implementación de un nuevo modelo de desarrollo, basado en la industrialización sustitutiva y con un fuerte intervencionismo estatal -que, paradójicamente implementarían los gobiernos autoritarios conservadores de la década del 30- y que se proyectaría hasta su crisis, en 1975 (Bernal-Meza, 2001; 2001ª). 
El nuevo modelo se había iniciado también con grandes expectativas. La Argentina lo comenzaba con una importante ventaja respecto de sus vecinos, contando con más de 50.000 establecimientos industriales, que representaban el 20\% del PBI (Kosacoff, 1993). Para 1950, la industria dominaba la generación de empleo, pero la proporción de exportaciones de manufacturas originadas en ese sector, respecto de la producción manufacturera total siguió siendo baja; el ingreso per cápita había descendido a 1/3 del norteamericano y a 1/2 del correspondiente a Europa occidental. Aún así, la producción per cápita respecto del Japón era 2:1 y de más de 3:1 en relación a Corea y Taiwán. Pero el liderazgo industrial y tecnológico siempre había quedado en manos de las corporaciones transnacionales, que para 1955 contribuían con el 20\% del producto, cifra que aumentaría al 1/3 para 1975. Para ese último año el PBI había descendido de 2/3 a 1/3 respecto del promedio de países industrializados, mientras que Japón ahora doblaba el PBI argentino y la diferencia respecto de Corea y Taiwán se había reducido en 1/3. Significativamente, la tasa de ahorro era más o menos del 20\% del PBI y comparable a la de los países líderes, mientras que el $99 \%$ de la formación de capital se había financiado con ahorro interno (Ferrer, 1998). Estas solas cifras y datos estadísticos debían echar por tierra los supuestos con los cuales el neoliberalismo iniciado en 1976 justificaría el nuevo modelo de inserción.

La evolución del modelo neoliberal marca dos períodos claramente diferenciables: 1976-1983 y 1989-2002. En el primero se sentaron las bases económicas, institucionales y normativas que permitirían poner al sector financiero en el liderazgo de la estructura de poder económico. Se desreguló el mercado de capitales, iniciándose la apertura comercial unilateral y hubo una transferencia neta de ingresos desde los sectores industrial y laboral hacia el sector financiero. En la segunda, bajo la presidencia de Carlos Menem, la apertura y desregulación del sector financiero y del comercio exterior se profundizó, acompañándose el proceso de una desregulación de los mercados laborales y de servicios, de la reforma del Estado y de la privatización de las empresas públicas, incluyendo industrias, servicios y bancos, que llevarían a la Argentina a una tasa de desempleo sin antecedentes históricos, superiores al 20\%. Ambos períodos se caracterizarían por déficits permanentes del comercio exterior y un aumento extraordinario del endeudamiento externo, el que de 3.000 millones de dólares a fines de 1975 se había multiplicado por 50 veces al comenzar el siglo XXI. 
En relación a la integración con Brasil, la llegada al gobierno de Carlos Menem marcó un nuevo giro, drástico, de profundización de las políticas de ajuste estructural y tanto en este país, como en Brasil, bajo las presidencias de Collor y después Cardoso, habría coincidencias en la adopción del modelo económico neoliberal. Este cambio se reflejó en la nueva concepción de la integración, bajo el paradigma del regionalismo abierto (Bernal-Meza, 1994; 2000) y que prevalecería durante los diez años del Mercosur.

Las esperanzas de cambio, con el triunfo de la Alianza (radicalismo-Frepaso) rápidamente se frustraron. De la Rúa no fue capaz de consolidar en una alianza políticosocial la fuerza necesaria para superar la crisis derivada de la implementación de tal modelo. Por el contrario, creyó ver en su continuidad -incluyendo la Convertibilidad- e integrando a su creador, Domingo Cavallo en su equipo ministerial, al frente de Economía, la solución a la crisis, reflejada en el crecimiento constante del desempleo y subempleo, de la continuidad de la recesión económica y del endeudamiento externo.

Como su antecesor, De la Rúa abandonó rápidamente todas sus promesas de campaña. Su fracaso, contribuyó extraordinariamente al descrédito de la clase política y, por tanto, del propio sistema, generando una profunda crisis de desconfianza en el funcionamiento de las instituciones, incluyendo la Justicia, sospechada de acompañar las políticas gubernamentales a cambio de garantías de permanencia en los cargos. Paralelamente, la recesión económica, arrastrada desde el año 1998 se acentuó, junto al crecimiento desorbitante del gasto público, las altas tasas de interés, la fuga de capitales y la debilidad de la figura presidencial, todo lo cual disminuyó extremadamente la capacidad de un gobierno que, por convicción tampoco se apartó del credo neoliberal. El avasallamiento de los derechos a la propiedad e igualdad, instrumentado a través de Decretos presidenciales (comienzos de diciembre de 2001) que impedían la libre disposición de los depósitos bancarios (plazos fijos, ahorros en cuentas a la vista, depósitos en cuentas corrientes) aceleró su caída. Su discurso astigmático determinó el desenlace de los acontecimientos. 
Duhalde asumió el gobierno como un presidente de transición, luego de conducir un movimiento de poder interno que virtualmente depuso al anterior presidente, designado por el Congreso y de su propio partido, Rodríguez Saa, ante la disyuntiva de renunciar o enfrentar la fuerza de su aparato de poder centrado en los populosos municipios del gran Buenos Aires. Falto de legitimidad popular-ciudadana, pero con el apoyo legislativo de gran parte del partido Justicialista, del Radicalismo y el Frepaso -alianza ésta que había abandonado el gobierno a fines de diciembre pasado- reuniendo así el apoyo de lo que se considera aquí como el segmento más despreciado de la política pública.

Si bien el mecanismo institucional por el que Duhalde fue electo presidente es el establecido en la Constitución para estos casos de crisis por acefalía del Ejecutivo; es de decir, es un presidente legítimo, carece en absoluto de legitimidad ante el conjunto de la sociedad. Para subsanar este vacío, actualmente está buscando apoyos y negociando con el poder sindical, sin tener en cuenta que éste carece también de la legitimidad de los trabajadores que son sus representados, pero que están abandonados a su suerte. En todo caso lo único que puede obtener Duhalde es más ilegitimidad de la que ya tiene.

En su primer discurso ante la Asamblea Legislativa prometió tres cosas que tranquilizaban a distintos sectores de la población: salir de la convertibilidad 1 a 1 de la moneda, devolver los depósitos de los ahorristas en las monedas en que éstos fueron hechos y poner la política pública al servicio de la recuperación del empleo, el apoyo a la producción y la asistencia social a los 14 millones de personas hoy excluidos totalmente del empleo, el consumo y la satisfacción por tanto de sus necesidades básicas más elementales de vida. De ello, hasta el presente -y nadie espera que esto cambie- no cumplió ninguna.

La primera era una declaración populista que crearía más problemas que los existentes con la convertibilidad; hecha sin el más mínimo análisis de sus efectos y consecuencias. La salida abrupta de la convertibilidad generó una corrida de precios y de desabastecimiento que llevó la inflación real acumulada -es decir aquella cuya diferencia deben pagar los ciudadanos con salarios depreciados sobre los cuales se mantienen reducciones acumuladas desde las administraciones anteriores- entre un 30 y un $40 \%$ en 
sólo dos meses. A partir de allí, un Plan Económico que se proponía una tasa de inflación anual menor al $15 \%$ era, simplemente, un engaño.

El segundo engaño fue la devolución de los depósitos. Es cierto que se estableció un mecanismo para el reintegro, en un plan que va de los diez años, para aquellos que aceptaran un bono de deuda, en un país en default, a los dos años, para los que optaran por la "pesificación", pero que representa para los ahorristas pérdidas en ningún caso menores al 70\% (en relación a la devaluación real de la moneda); esto siempre y cuando en el futuro la economía se mantuviera en márgenes manejables, lo contrario, que es el único escenario previsible, es que esos depósitos se licuarán, constituyendo la más grande expropiación de propiedad realizada por gobierno alguno desde las experiencias de la Rusia soviética.

El tercero, que los sectores de la producción y los desposeídos serían los destinatarios de una nueva política económica y social.

\section{Las alternativas de las relaciones bilaterales y el Mercosur frente a la crisis argentina del 2002}

El fracaso del modelo neoliberal aplicado casi sistemáticamente en Argentina durante los últimos veinticinco años, el derrumbe de su economía, la crisis política y de legitimidad de sus instituciones obligan también a revisar toda la naturaleza del Mercosur.

El mecanismo financiero actualmente en vigencia en ese país, llamado "Corralito", que fuera implantado por Cavallo antes de salir junto al gobierno de De la Rúa, fue profundizado por Duhalde. El mismo ha implicado graves restricciones al retiro de depósitos en cuentas corrientes, depósitos a plazo fijo y al retiro de depósitos salariales, lo que ha profundizado gravemente el proceso recesivo de la economía argentina, que ya se arrastraba por cuatro años; agravando también el déficit fiscal, ante la caída de impuestos por consumo. 
El nuevo tipo de cambio, con una devaluación técnica del 29\% y una real de más del $70 \%$, ahora protege a la producción nacional. Pero el problema no radica en esto, sino en que hoy no hay consumo en la Argentina, por tanto, las exportaciones con destino a este país, así como la producción nacional no tienen mercado demandante.

Durante los últimos diez años 150.000.000.000 de dólares se fugaron y dejaron un país sin recursos financieros. Ni siquiera sus empresas tradicionales son nacionales. Argentina no tiene caminos, aviones, barcos ni trenes propios. De las 500 grandes empresas existentes 300 son extranjeras; éstas ganan cuatro veces más que las locales, pero invierten en el país sólo 1/4 de lo que invierten las empresas nacionales. Así, las herramientas del desarrollo deben ser reformuladas. Paralelamente, las empresas privatizadas no se han visto perjudicadas por la devaluación porque todos sus dividendos fueron sacados del país con anterioridad a las medidas de pesificación y control de cambios y, además, se han visto beneficiadas con la pesificación de sus deudas (1 dólar por 1 peso). Por último, siguen obteniendo beneficios con los aumentos de tarifas, en el ámbito de los servicios públicos, que no se condicen con la realidad, pero concertados mediante lobbies con la clase política.

La crisis estructural del desarrollo argentino, por tanto, no puede explicarse por el paradigma "centro-periferia" y el deterioro de los términos de intercambio, ni por el proteccionismo del Norte, aunque esas condiciones hayan ayudado; pero nunca fueron las determinantes, como sí ocurrió en muchos otros países del mundo en desarrollo (BernalMeza, 2001).

Ante este escenario ¿cuál es el pronóstico? En primer lugar, hay que comprender que, como consecuencia de la implantación del modelo neoliberal más extremo de los que se conocen en América Latina, cuya expresión de "capitalismo salvaje" debe ser aplicado para los períodos de los gobiernos de Menem, De la Rúa y Duhalde, la Argentina ha quedado al borde de la anarquía. Hay muchos analistas que hablan de una eventual guerra civil si llegaran a fracasar las políticas del actual gobierno. No creo que haya una guerra civil, simplemente por el hecho de que no hay dos modelos opuestos de Estado y Sociedad que hoy confronten en la sociedad argentina, pero sí es evidente que un nuevo 
modelo de desarrollo nacional debe ser discutido en este país. Es en ese contexto donde la integración del Mercosur debe encontrar también su nuevo destino (Bernal-Meza, 2002).

Entre las consecuencias internas a nivel social (pero que repercutirán sin dudas sobre el tejido socio-territorial de una zona "mercosur"), el desmembramiento de las familias y de los lazos de los afectos es evidente. Oficialmente se van del país entre 700 y 800 argentinos por día. El impacto de este nuevo fenómeno hay que considerarlo dentro del contexto de un país en donde la idiosincrasia hace que los efectos nocivos sean muy marcados.

No hay distinción de clases ni de conocimientos entre los que abandonan el país; sin embargo, la emigración se presenta sobre todo entre los jóvenes. Uno de los factores principales es el descreimiento generalizado de encontrar una solución para el país y por lo tanto un futuro personal y familiar en esta tierra.

A nivel emocional se han producido diferentes abusos (sociales, políticos, económicos) que no encuentran contención en las estructuras de estas latitudes. Las consecuencias negativas de lo desconocido (el nuevo país de acogida) no superan -al menos en teoría- las ya experimentadas en Argentina, porque las personas ya no soportan más abusos, los que comienzan con las políticas públicas. La sensación de "estar abusado" será una de las nuevas enfermedades psiquiátricas del futuro próximo.

Es optimista tomar en cuenta el hecho que el comportamiento de las economías de Brasil y de Chile frente a la debacle -los dos países que más estrechamente están vinculados a la economía argentina en la región- señala que los riesgos de extrapolación o internacionalización de la crisis argentina son menores a lo que se preveía. Este es también un elemento importante que rescata el valor de los instrumentos de política económica y monetaria en manos de los gobiernos. No obstante, deberá tenerse presente que por la nueva situación de crisis que vive la Argentina, con un programa económico sin definición, el inicio de la armonización y coordinación macroeconómica será postergado, ya que las 
metas fiscales y de inflación argentinas serán una incógnita al menos hasta transcurrido gran parte del año 2002.

Argentina requerirá de una rápida expansión de su comercio de exportación, fuente de recursos financieros genuinos. Las condiciones están dadas hoy para profundizar la integración del Mercosur y por una renegociación del sector agrícola en la OMC; pero ésta sólo será posible a través de la concertación de políticas entre los países en desarrollo. Un MERCOSUR sólido, unificado en sus políticas externas, con capacidad de negociación, puede obtener ventajas de una negociación con la Unión Europea, las que luego podrían extrapolarse a la OMC a través de una concertación con el Grupo Cairns, o también a través del mecanismo inverso. Así, ambos escenarios de acción conducen al fortalecimiento de la alianza argentino-brasileña y de la sociedad 4+2. He allí el desafío, que enfrenta además el dilema de que nadie quiere en estos momentos negociar con Argentina.

El nuevo punto de inflexión, que se produjo con la renuncia de De la Rúa y la llegada al gobierno de Eduardo Duhalde, pone al Mercosur y, en particular a las relaciones bilaterales argentino-brasileñas, que constituyen su eje estratégico, en un momento de expectativas. La eliminación de la Convertibilidad de la moneda argentina y la reducción del perfil de prioridad en las relaciones de Buenos Aires con Estados Unidos -puesto de manifiesto en el hecho que el primer viaje al exterior del canciller Carlos Ruckauf fue justamente a Brasilia- suponen la desaparición de los dos "problemas" que el gobierno brasileño permanentemente planteó como las grandes dificultades para profundizar esa alianza estratégica entre ambos países y hacer avanzar así al proceso de integración. La propia situación de crisis que vive Argentina debería llevar también a considerar al Mercosur como un ámbito clave de cooperación solidaria. Desde este punto de vista, Brasil tiene una óptima posibilidad para demostrar su compromiso político y económico con la integración. El reto para la última parte de la gestión presidencial de Cardoso es también crear las condiciones para una relación estratégica, asumiendo el desafío del liderazgo subregional, que debería reflejarse en políticas activas de apoyo para la Argentina, más allá de las palabras. 


\section{Conclusiones y perspectivas}

Los argentinos tienen hoy un presidente, que rige sus vidas y su destino, pero que la sociedad nunca votó. Duhalde es la continuidad de una alianza entre el gran capital financiero, los grandes grupos económicos monopólicos y los políticos, que se entronizó en la vida argentina luego del retorno de la democracia en 1983, con algunos agravantes que los inversores, internos y externos deberán tener en cuenta para sus estrategias presentes y futuras: no existe un Estado de derecho que garantice hoy la propiedad privada, es decir que el principio contractual que justificó este tipo particular de Estado occidental moderno, la asociación de capitalismo y ordenamiento jurídico-político-administrativo, sostenido por la construcción ideológica del Leviatán de Hobbes, no existe. El Estado, por mano de sus administradores gubernamentales, se ha apropiado de la propiedad de los ahorristas y sus 68.000.000.000 dólares depositados en los bancos, recursos que sostuvieron la fuga de capitales por la cual la sociedad entre bancos y grandes grupos económico-financieros sacaron del país 28.000 millones de dólares en unos cuantos meses. Aún más, a través de meros decretos, el gobierno desplazó al Poder Judicial de su potestad para responder ante los ciudadanos por la defensa de sus derechos garantizados por la Constitución, como son la protección de la propiedad y la posibilidad de recurrir a la justicia.

El segundo instrumento para continuar con la apropiación y transferencia de riqueza desde la sociedad civil hacia los grandes grupos económico-financieros ha sido la normativa por la cual se pesificaron las deudas en dólares de éstos. Aduciendo la defensa de los deudores constituidos por las pequeñas y medianas empresas y salvar el patrimonio de aquellos que compraron una vivienda con una hipoteca según la ley de Convertibilidad, se ha permitido que los grandes grupos económicos, constituidos por alianzas entre bancos, empresas filiales, bancos internacionales y empresas privatizadas, paguen a valor 1 peso $=$ 1 dólar sus deudas. El sistema tan perverso se explica de la siguiente manera: una empresa (como de las que existen decenas de ejemplos) tomó créditos en dólares, en la plaza local, por 400, 600 o más millones de dólares, que luego transfirió al exterior a través de esos mismos bancos que les prestaron y que hoy, con un tercio de esos recursos pagarán sus créditos, vendiendo los dólares en el mercado local a una cotización de 1 dólar = 3 pesos. 
El costo para el pueblo argentino de este enriquecimiento ilícito, según las estimaciones más sobrias alcanza los 22.000 millones dólares, cifra que nuevamente pagará el pueblo argentino, porque engrosarán la deuda externa.

La última esperanza para la continuidad del actual gobierno está en el éxito de la gestión de Roberto Lavagna al frente del ministerio de Economía. Con él se espera también un relanzamiento de las relaciones con Brasil y un retorno de Argentina hacia el escenario del Mercosur como una de sus prioridades, aún cuando la dependencia financiera de Argentina, derivada de su opción por una inserción en el mercado internacional de capitales, la obliga a mantener una estrecha relación con el FMI y, por tanto, con su principal decisor, Estados Unidos.

En el "Prólogo" que Lavagna escribió para mi último libro ${ }^{6}$-abordando el problema de la globalización- señalaba que frente a las alternativas del "seguidismo", reflejadas en la visión de lo inevitable de un proceso en el cual los Estados dejan de tener vigencia como sujeto de las relaciones internacionales; de la negación aislacionista, que ve como única alternativa una economía y un trabajo nacional protegidos por barreras anti-importación y/o anti-inversión; o frente a la visión pragmática que clama por la primacía de lo nacional cuando se trata de juzgar las violaciones a los derechos humanos, pero que en materia de pensamiento económico apoyan la más completa declinación de cualquier margen para la acción nacional o aún regional (Mercosur), era más correcto reconocerla, siguiendo el camino de una adaptación activa, rechazando la resignación de una adaptación simplemente pasiva. Advierte sobre los riesgos del "pensamiento único" y los graves efectos de las políticas de ajuste y cambio estructural. Concluía que "el creciente número de excluidos dentro de los países y entre los países ha puesto en tela de juicio la teoría del derrame y está convocando a desarrollar un pensamiento complejo que unifique la liberación de las fuerzas del mercado y los equilibrios sociales, territoriales, ambientales e inter-generacionales"

\footnotetext{
${ }^{6}$ Sistema Mundial y Mercosur. Globalización, Regionalismo y Políticas Exteriores Comparadas, Buenos Aires, Nuevohacer/Grupo Editor Latinoamericano y Universidad Nacional del Centro de la Provincia de Buenos Aires, 2000.

${ }^{7}$ Roberto Lavagna, en el Prólogo a Sistema Mundial y Mercosur, op. cit., pp. 16-17.
} 
Esta toma de posición frente a las visiones maximalistas sobre la mundialización/globalización permiten percibir que su visión está más bien lejos del "pensamiento único" que ha inspirado las políticas de ajuste estructural a partir del llamado Consenso de Washington y dan a los proyectos estratégicos de integración regional una mejor alternativa. Lavagna es un impulsor de una "integración de contenido estratégico y estructural", como la que hemos defendido en nuestros estudios ${ }^{8}$. En reciente documento señaló que "o instrumento tarifário isoladamente, na ausência de outros mecanismos específicos e/ou de políticas macroeconômicas coordenadas, mostrou toda a sua incapacidade de levar o processo adiante. Ficou claro que se havia confundido uma condição necessária - no plano tarifário - com as condições suficientes requeridas por um projeto estratégico de integração. Não se constrói um mercado comum sem um projeto e a partir de um único instrumento (...). O resultado destes erros de concepção tem sido não somente a desaceleração do processo de integração, mas também a "reprimarização" paulatina das exportações argentinas intra-regionais, concomitantemente ao fato de o Brasil seguir adquirindo um papel predominantemente industrial." (Lavagna, 2002), palabras que expresan una visión crítica sobre el modelo de integración elegido para el Mercosur y seguido durante la reciente década.

Así, respecto de las relaciones bilaterales argentino-brasileñas y para el futuro del Mercosur, es esperanzadora la posición favorable a ambos destinos externos de su país que tiene el nuevo ministro de Economía argentino. No obstante, creo que dos factores condicionan las perspectivas: en primer lugar, la resolución de la crisis argentina en sus aspectos más cruciales, vinculadas a la reconstrucción de su institucionalidad, la recuperación de la credibilidad pública, el respeto a la ley y la Constitución, la solución al “corralito” y la devolución de los depósitos y la recuperación mínima de la actividad económica; en segundo lugar, la decisión del gobierno brasileño de transformar en hechos la declamada "solidaridad del Mercosur".

\footnotetext{
${ }^{8}$ Cfr. Raúl Bernal-Meza, América Latina en la Economía Política Mundial (Buenos Aires, Grupo Editor Latinoamericano, 1994) y Sistema Mundial y Mercosur, op. cit.
} 
Debe comprenderse entonces que la Argentina no sólo necesita un nuevo modelo de desarrollo sino de Estado y sociedad, incluyendo el cambio de sus instituciones. De allí que hablemos de una "crisis terminal". La esperanza, es que aún cuenta con un capital humano importante, por su educación y cultura.

\section{Bibliografía}

BAUMANN, Renato, Mercosul: Origens, Ganhos, Desencontros e Perspectivas, in: BAUMANN, Renato (org), MERCOSUL. Avanços e Desafios da Integração. Brasília: IPEA/CEPAL, 2001, p. 19-68.

BERNAL-MEZA, Raúl. América Latina en la Economía Política Mundial. Grupo Editor Latinoamericano: Buenos Aires, 1994. Sistema Mundial y Mercosur. Globalización, Regionalismo y Políticas Exteriores Comparadas. Nuevohacer-Grupo Editor Latinoamericano/Universidad Nacional del Centro de la Provincia de Buenos Aires: Buenos Aires, 2000.

Argentina. La crisis del desarrollo y de su inserción internacional. São

Paulo, Centro de Estudos Fundação Konrad Adenauer/Programa de Estudos Europeos, Universidade Federal do Rio de Janeiro, Análisis e Informaciones, n 1, Mayo de 2001.

Argentina: ¿crisis coyuntural o estructural. In: Carta Internacional.

USP-Política Internacional nº. 99, año IX: São Paulo, 2001, págs. 7-10.

Os dez anos de MERCOSUR e a crise Argentina. In: Política Externa,

São Paulo, Paz e Terra/Gacint-USP-Universidade de São Paulo/ Instituto de Estudos

Econömicos e Internacionais, vol. 10, $\mathrm{n}^{\circ}$ 4, março-abril-maio 2002, págs. 7-46.

FERRER, Aldo. Hechos y ficciones de la globalización. Argentina y el MERCOSUR

en el sistema mundial. Fondo de Cultura Económica: Buenos Aires, 1998.

KOSACOFF, Bernardo, et. al., El Desafio de la Competitividad. La Industria

Argentina en Transformación. Alianza Editorial/CEPAL, Buenos Aires, 1993.

LAVAGNA, Roberto. Os Desafíos do MERCOSUR. In: Revista Brasileira de

Comercio Exterior. Brasil, 4 de abril de 2002.

RAPOPORT, Mario. Historia Económica, Política y Social de la Argentina (1880-

2000). Ediciones Macchi, Buenos Aires, 2000. 
TREBER, Salvador. La Economía Argentina Actual: 1970-1987. Buenos Aires, 1987. 